\title{
Coupled mode analysis of the photonic crystal waveguide
}

John M. Choi, Yong Xu, Amnon Yariv

John M. Choi, Yong Xu, Amnon Yariv, "Coupled mode analysis of the photonic crystal waveguide," Proc. SPIE 5000, Photonic Crystal Materials and Devices, (9 July 2003); doi: 10.1117/12.480061

SPIE Event: Integrated Optoelectronics Devices, 2003, San Jose, CA, United States 


\title{
Coupled mode analysis of the photonic crystal waveguide
}

\author{
John M. Choi, Yong Xu, and Amnon Yariv \\ California Institute of Technology, Caltech 136-93, Pasadena, CA, 91125
}

\begin{abstract}
Starting from an intuitive picture of photons bouncing back and forth within a slab of a uniform medium surrounded by photonic crystal (PC) layers, we develop a coupled mode formalism for the analysis of a PC waveguide. The modal solution in the core is given by counter-propagating waves while the cladding field is derived from a modified coupled mode formalism. This coupled mode approach is used to analyze the dispersion and loss of the PC waveguide. Although coupled mode theory is usually applied to structures with a small index contrast perturbation, we find that our coupled mode formalism with an empirical coupling constant can be used to model a large index contrast PC waveguide. A single constant is used to analyze the dispersion and the loss of a two dimensional PC waveguide, a GaAs substrate with air holes. Our results are corroborated with a two dimensional finite-difference time-domain (FDTD) simulation and we find good quantitative agreement between the coupled mode theory and the FDTD analysis.
\end{abstract}

Keywords: photonic crystal waveguide, coupled mode theory, propagation loss, numerical analysis

\section{INTRODUCTION}

Photonic crystal waveguides have received much attention as a promising candidate for compact integrated planar lightwave circuits. ${ }^{1-4}$ However, the analysis and design of these waveguides is performed through the timeconsuming process of numerically solving Maxwell's equations. These methods generally fall into two categories, the finite difference time domain ${ }^{5}$ (FDTD) and plane wave expansion methods. ${ }^{6-8}$ The FDTD method is relatively easy to implement, but is computationally intensive while the plane wave method is restricted to purely periodic structures. Both methods are valuable tools in the study of photonic crystal structures, but the numerical analysis can be time consuming and the numerical approaches give little physical intuition.

By using coupled mode theory, we can obtain an analytic model of the field propagtaion. ${ }^{9}$ This approach is based on an intuitive picture of a photon bouncing back and forth between two photonic crystal layers. The mode in the core is assumed to consist of counter propagating waves and the modes in the cladding are given by coupled mode equations. Solving these equations results in the full transverse field distribution as well as the dispersion and propagation loss. By comparing to 2D FDTD simulations, we see that there exists a regime when the coupled mode formalism is valid and can provide quantitatively accurate results. Thus we can gain greater intuitive understanding that can help us in predicting the properties of photonic crystal waveguides.

\section{COUPLED MODE THEORY}

The two dimensional geometry analyzed is shown in Fig. 1. The photons are confined in the waveguide core by the Bragg reflection off the 2D grating. Under the condition of small index perturbation, the solution satisfies the Helmholtz equation

$$
\nabla^{2} \mathbf{E}+\mu \epsilon(\mathbf{r}) \omega^{2} \mathbf{E}=0
$$

We assume the guided modes take the form of forward and backward propagating plane waves in the transverse direction and the propagation constant is allowed to be complex, $\beta=\beta_{R}+i \beta_{I}$, in order to describe the propagation loss.

$$
\mathbf{E}(\mathbf{r}, t)=E(x) E(z, t)=\left(A(x) e^{i k x}+B(x) e^{-i k x}\right) e^{i \omega t-i \beta z}
$$

where

$$
k=\left\{\begin{array}{cl}
k_{b}=\frac{2 \pi}{b}, & \text { cladding } \\
k_{R}=\sqrt{\bar{\epsilon} \frac{\omega^{2}}{c^{2}}-\beta_{R}}, & \text { core }
\end{array}\right.
$$


where $\bar{\epsilon}$ is the average dielectric constant of the photonic crystal cladding. While this method can be applied to lossy/active structures, ${ }^{10}$ we restrict ourselves to a structures with no loss/gain in the material. Since the index variation in the cladding is periodic, we can rewrite it as

$$
\begin{gathered}
\Delta \epsilon(\mathbf{r})=\sum_{l, m} \Delta \epsilon_{l m} e^{i\left(l k_{b} x+m k_{a} z\right)} \\
\Delta \epsilon_{l m}=\frac{1}{a b} \int_{a b} \Delta \epsilon(\mathbf{r}) e^{-i\left(l k_{b} x+m k_{a} z\right)} \mathrm{d} x \mathrm{~d} z
\end{gathered}
$$

Substituting Eq. 2 into Eq. 1 and using the slowly varying approximation, $\left|\partial^{2} / \partial x^{2}\right| \ll|2 k \partial / \partial x|$, we obtain

$$
i 2 k \frac{\partial}{\partial x} A(x)+\left(k_{R}^{2}-k^{2}\right) A(x)-i 2 \beta_{R} \beta_{I} A(x)=-\frac{\omega^{2}}{c^{2}} B(x) \frac{1}{a b} \int_{0}^{a} \int_{0}^{b} \Delta \epsilon(\mathbf{r}) e^{-i 2 k x} \mathrm{~d} x \mathrm{~d} z
$$

where $a b$ is the area of the unit cell. We also make the following definitions:

$$
\begin{aligned}
\gamma & =-\frac{\beta_{R} \beta_{I}}{k} \\
\kappa & =i \frac{k_{0}^{2}}{2 \bar{\epsilon} k} \frac{1}{a b} \int_{a b} \Delta \epsilon(\mathbf{r}) e^{-i 2 k x} \mathrm{~d} x \mathrm{~d} z
\end{aligned}
$$

where we have defined $k_{0}=\sqrt{\bar{\epsilon}} \omega / c$. With these definitions, and similar manipulations for $B(x)$, we get the coupled mode equations in matrix form

$$
\frac{\partial}{\partial x}\left[\begin{array}{l}
A(x) \\
B(x)
\end{array}\right]=\left[\begin{array}{cc}
-(\gamma-i \Delta k) & \kappa \\
\kappa^{*} & (\gamma-i \Delta k)
\end{array}\right]\left[\begin{array}{c}
A(x) \\
B(x)
\end{array}\right]
$$

where $\Delta k \equiv k_{R}-k$. Solving this set of equations results in

$$
\left[\begin{array}{l}
A(L) \\
B(L)
\end{array}\right]=\left[\begin{array}{cc}
-\frac{(\gamma-i \Delta k)}{S} \sinh (S L)+\cosh (S L) & \frac{\kappa}{S} \sinh (S L) \\
\frac{\kappa^{*}}{S} \sinh (S L) & \frac{(\gamma-i \Delta k)}{S} \sinh (S L)+\cosh (S L)
\end{array}\right]\left[\begin{array}{l}
A(0) \\
B(0)
\end{array}\right]
$$

where

$$
S=\sqrt{|\kappa|^{2}+(\gamma-i \Delta k)^{2}}
$$

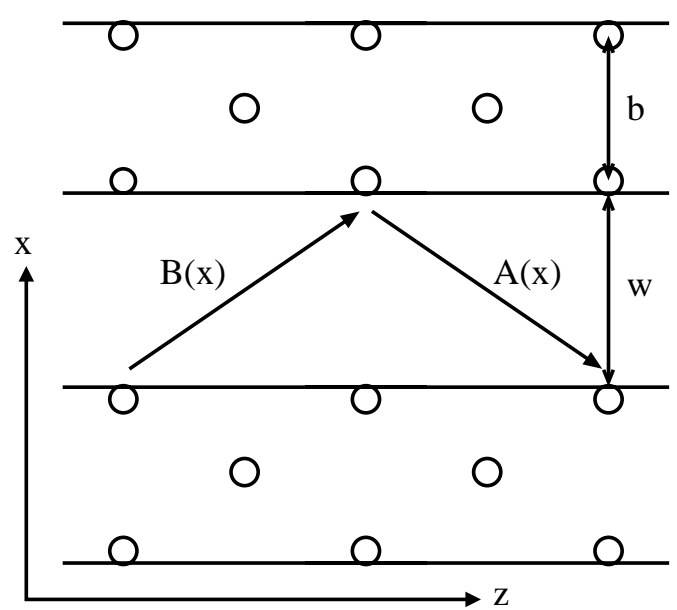

Figure 1. A two dimensional triangular lattice photonic crystal waveguide. The waveguide core width is $W$. The cladding thickness is equal to one unit cell, $b$, for illustrative purposes. The forward, $B(x)$, and backward, $A(x)$, traveling waves are shown reflecting off the cladding. 
In the homogenous core with $\kappa=0$, this reduces to

$$
\left[\begin{array}{l}
A(L) \\
B(L)
\end{array}\right]=\left[\begin{array}{cc}
e^{-\gamma L} & 0 \\
0 & e^{\gamma L}
\end{array}\right]\left[\begin{array}{l}
A(0) \\
B(0)
\end{array}\right]
$$

At the interface between the core and cladding, we require that the field and its derivative be continuous. Using the notation shown in Fig. 2, we get the following relation

$$
\left[\begin{array}{l}
A_{1}(W / 2) \\
B_{1}(W / 2)
\end{array}\right]=\frac{1}{2}\left[\begin{array}{cc}
\left(1+\frac{k_{0}}{k_{1}}\right) e^{i k_{0} W / 2} & \left(1-\frac{k_{0}}{k_{1}}\right) e^{-i k_{0} W / 2} \\
\left.1-\frac{k_{0}}{k_{1}}\right) e^{i k_{0} W / 2} & \left(1+\frac{k_{0}}{k_{1}}\right) e^{-i k_{0} W / 2}
\end{array}\right]\left[\begin{array}{c}
A_{0}(W / 2) \\
B_{0}(W / 2)
\end{array}\right]
$$

where $k_{0}$ is $k_{R}$ from Eq. 3 in the core, and $k_{1}$ is the corresponding transverse $k$ vector in the cladding, $k_{b}$. From Eqs. 10, 12, and 13, we can construct a matrix relation for the field at the boundary, $A_{2}$ and $B_{2}$, in relation to the field in the core, $A_{0}$ and $B_{0}$.

$$
\left[\begin{array}{l}
A_{2} \\
B_{2}
\end{array}\right]=T\left[\begin{array}{l}
A_{0}(0) \\
B_{0}(0)
\end{array}\right]=M_{1} M_{10} M_{0}\left[\begin{array}{l}
A_{0}(0) \\
B_{0}(0)
\end{array}\right]
$$

where $M_{0}$ is the matrix from Eq. $12, M_{1}$ is the matrix from Eq. 10, and $M_{10}$ is the interface matrix from Eq. 13. The boundary condition is obtained by observing that the incoming field at the outer boundary, $A_{2}$ in Fig. 2, should be zero and by taking advantage of the symmetry of the waveguide along the $z$ axis. The waveguide modes can then be divided into even and odd modes where $A_{0}(0)=B_{0}(0)$ for the even mode and $A_{0}(0)=-B_{0}(0)$ for the odd mode. The matrix $T$ is a function of the complex $\beta$. For a mode with a given symmetry, its complex propagation constant $\beta$ is given by the condition of $A_{2}=0$.

After obtaining the complex propagation constant, the transverse field distribution is given by

$$
E(x)=E_{0}\left(e^{\left(-\gamma+i k_{R}\right) x}+e^{\left(\gamma-i k_{R}\right) x}\right), 0 \leq x \leq \frac{W}{2}
$$



Figure 2. The waveguide shown with the counter propagating fields from the coupled mode formalism in each layer. The $n=0$ layer corresponds to the core The $n=1$ layer is the cladding with thickness $L$. The $A_{2}$ and $B_{2}$ represent the field beyond the finite cladding layer. $B_{2}$ is the field leakage from the waveguide and accounts for the waveguide loss. $A_{2}$ is set to be zero since there should be no incoming field incident on the cladding. 
in the homogenous core, and

$$
\begin{aligned}
E\left(x^{\prime}=x-\frac{W}{2}\right)= & E_{0}\left(\left(C_{1} \cosh \left(S x^{\prime}\right)+C_{2} \sinh \left(S x^{\prime}\right)\right) e^{i k_{b} x^{\prime}}\right. \\
& \left.+\left(D_{1} \cosh \left(S x^{\prime}\right)+D_{2} \sinh \left(S x^{\prime}\right)\right) e^{-i k_{b} x^{\prime}}\right), x>\frac{W}{2}
\end{aligned}
$$

in the cladding and

$$
\begin{aligned}
\Gamma_{0} & =\gamma-i k_{R} \\
\Gamma_{1} & =\gamma-i \Delta k \\
C_{1} & =\cosh \left(\Gamma_{0} \frac{W}{2}\right)-\frac{k_{R}}{k_{b}} \sinh \left(\Gamma_{0} \frac{W}{2}\right) \\
C_{2} & =\frac{1}{S}\left(\left(\kappa-\Gamma_{1}\right) \cosh \left(\Gamma_{0} \frac{W}{2}\right)+\left(\kappa+\Gamma_{1}\right) \frac{k_{R}}{k_{b}} \sinh \left(\Gamma_{0} \frac{W}{2}\right)\right) \\
D_{1} & =\cosh \left(\Gamma_{0} \frac{W}{2}\right)+\frac{k_{R}}{k_{b}} \sinh \left(\Gamma_{0} \frac{W}{2}\right) \\
D_{2} & =\frac{1}{S}\left(\left(\kappa^{*}+\Gamma_{1}\right) \cosh \left(\Gamma_{0} \frac{W}{2}\right)-\left(\kappa^{*}-\Gamma_{1}\right) \frac{k_{R}}{k_{b}} \sinh \left(\Gamma_{0} \frac{W}{2}\right)\right)
\end{aligned}
$$

\section{PHOTONIC CRYSTAL WAVEGUIDE ANALYSIS}

In this section we apply the coupled mode formalism to analyze the dispersion, loss and field distribution of guided modes in a triangular lattice PC waveguide. For the PC waveguide shown in Fig. 3, according to Eq. 4 and Eq. 8 we have,

$$
\kappa=i \frac{k_{0}^{2}}{2 \bar{\epsilon} k_{b}} \sum_{l, m} \frac{1}{a b} \int_{a b} \Delta \epsilon_{l m} e^{i(l-2) k_{b} x+i m k_{a} z} \mathrm{~d} x \mathrm{~d} z
$$

We see that the most significant contribution should result when the forward and backward propagating modes are phase-matched $(l=2, m=0)$ so we choose to ignore all terms except $\Delta \epsilon_{20}$. Using Eq. 5 and 23 and

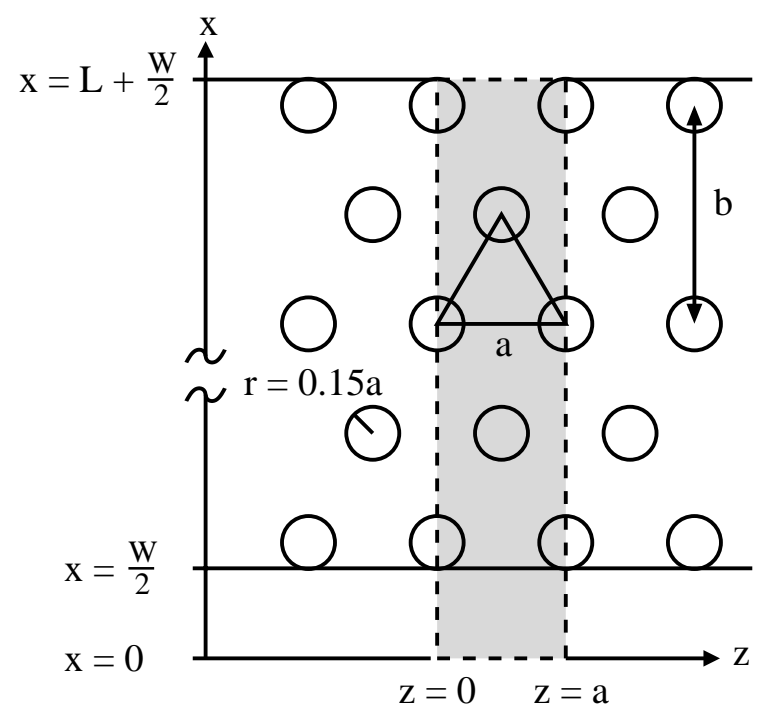

Figure 3. The 2 dimensional triangular lattice analyzed. The shaded region shows the equivalent domain simulated by the $2 \mathrm{D}$ FDTD simulation. The boundary conditions used are perfectly-matched layer at $x=L+W / 2$, even symmetry at $x=0$, and the Bloch boundary condition, $E(z=a)=E(z=0) \exp (i \beta a)$. 


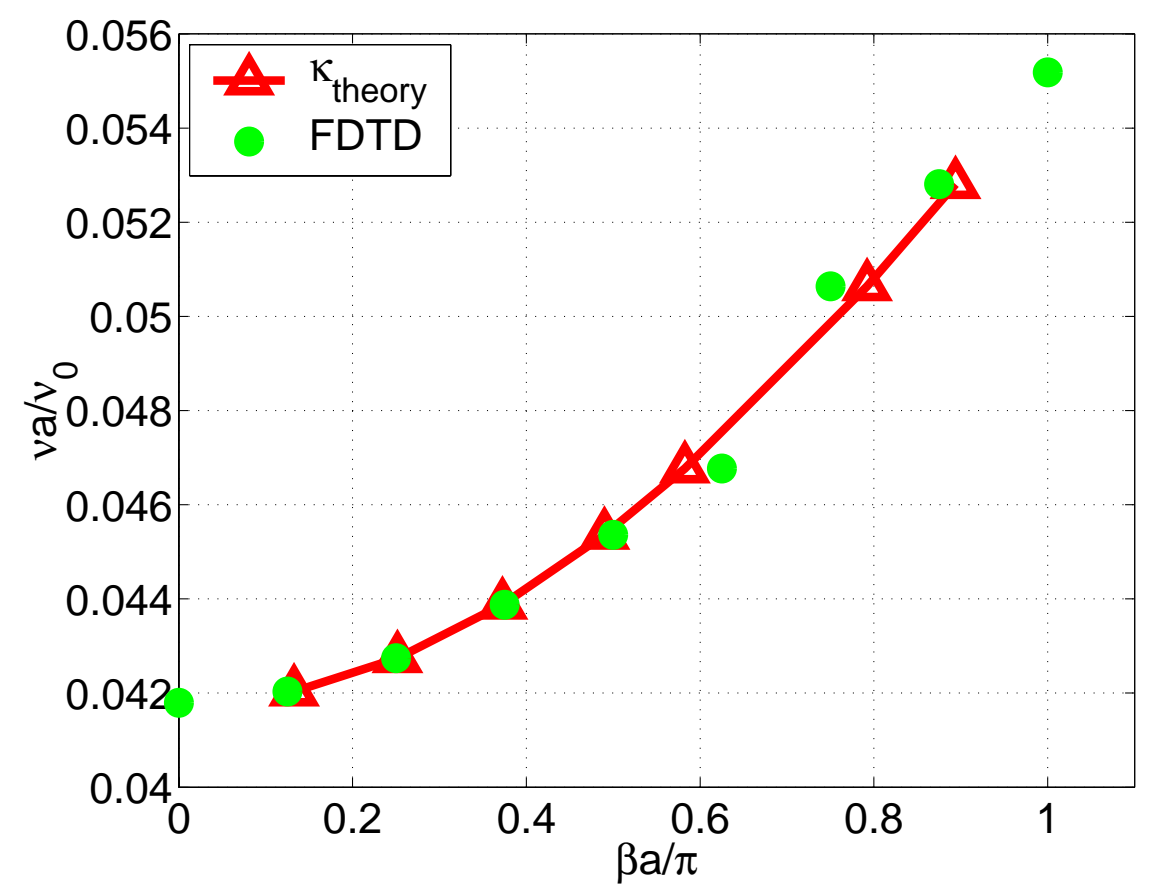

Figure 4. Dispersion of a GaAs slab, air hole, 2D triangular lattice photonic crystal waveguide. The filled circles show the $2 \mathrm{D}$ FDTD results. The triangles show the dispersion calculated using Eq. 24. This theory matches the FDTD results well for small propagation constants when $k_{0} \sim k_{b}$.

assuming that the holes are small, $A_{\text {hole }} \ll A_{\text {cell }}$, and restricting ourselves to the first order Bragg resonance which produces the strongest reflection, $\left|k_{b}\right| \sim\left|k_{0}\right|$, we then get

$$
\begin{aligned}
\kappa & =i \frac{k_{0}^{2}}{2 \bar{\epsilon} k_{b}} \frac{1}{a b} \int_{a b}\left(\epsilon_{\text {hole }}-\epsilon_{\text {slab }}\right) A_{\text {hole }}\left(\delta(x) \delta(z)+\delta\left(x-\frac{b}{2}\right) \delta\left(z-\frac{a}{2}\right)\right) e^{-i 2 k_{b} x} \mathrm{~d} x \mathrm{~d} z \\
& \sim i k_{b} \frac{A_{\text {hole }}}{A_{\text {cell }}} \frac{\left(\epsilon_{\text {hole }}-\epsilon_{\text {slab }}\right)}{\bar{\epsilon}}=i \frac{2 \pi^{2}}{3 a}\left(\frac{r}{a}\right)^{2} \frac{\left(n_{\text {hole }}^{2}-n_{\text {slab }}^{2}\right)}{\bar{n}^{2}}
\end{aligned}
$$

The assumptions leading to Eq. 24 can be applied when the index contrast is small. Typically, an index perturbation of the order of $\Delta n \sim 0.01-0.001$ is necessary for the coupled mode theory to be valid. However, we will apply it to the structure shown in Fig. 3 consisting of a GaAs substrate with air holes, $\Delta n \sim 2.6$. The triangular lattice is chosen so that $r=0.15 a$ and $b=\sqrt{3} a$. The small hole radius is chosen so that the guiding in the waveguide core is not due to total-internal-reflection. ${ }^{11,12}$ As a consequence, although we are looking for modes of a photonic crystal waveguide, there is no photonic band gap.

For the 2D FDTD, the computational domain is shown by the shaded region in Fig. 3. The boundary conditions consist of a perfectly matched layer $^{13}$ (PML) at $x=L+W / 2$, even symmetry at $x=0$, and the Bloch boundary condition such that $E(z=a)=E(z=0) \exp (i \beta a)$. The eletric field component out of the plane, $E_{y}$, corresponding to a TM mode is used for comparison with the scalar field from the coupled mode formalism.

We will only consider the lowest order even mode and analyze the case of a waveguide core equal to $W=$ $b / 4 .{ }^{9}$ Solving Eq. 14 using the coupling constant given by Eq. 24 yields the dispersion shown in Fig. 4 for a waveguide with 10 layers of cladding, $L=10 b$. The dispersion calculated by the 2D FDTD method is also shown for comparison. We find that the dispersion is not very sensitive to changes in $\kappa$, and thus the coupled mode approach shows good quantitative agreement with the FDTD. However, the propagation loss is highly dependent on $\kappa$. Although there is good qualitative agreement, the loss differs quantitatively. By

148 Proc. of SPIE Vol. 5000 


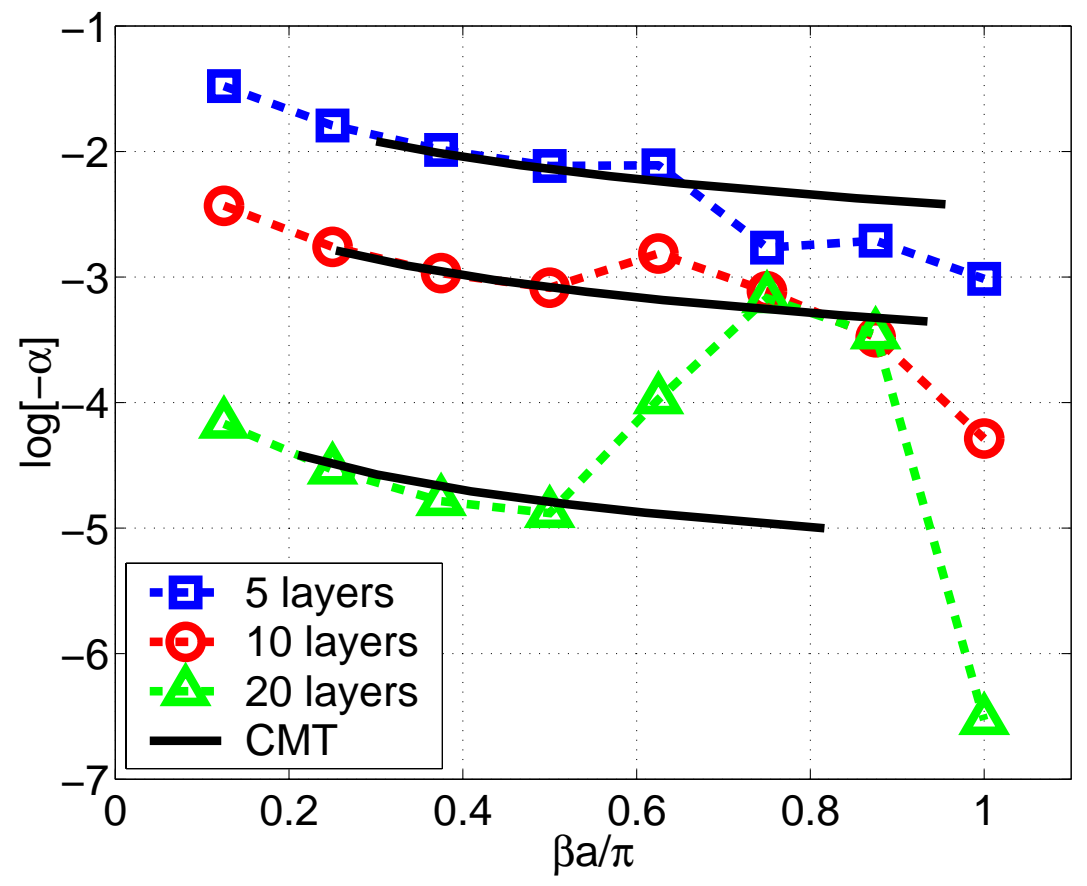

Figure 5. Propagation loss of photonic crystal waveguide. The loss is calculated for a GaAs substrate with air holes. Cladding widths of 5, 10, and 20 layers were simulated using 2D FDTD. The loss was calculated from the Q using Eq. 27. The loss was also predicted using coupled mode theory (CMT) from the imaginary part of the complex propagation constant using the same empirical coupling constant, $\kappa_{f i t}$, for all three waveguides.

using an empirical coupling constant, $\kappa$, we are able to obtain good quantitative agreement. In this case, the theoretical coupling constant was $\left|\kappa_{\text {theory }}\right|=0.0708$ whereas the empirical coupling constant was estimated to be $\left|\kappa_{\text {fit }}\right|=0.00452$. Although the difference is significant, around $30 \%$, this single empirical constant is sufficient to describe quantitatively the loss for almost all the guided modes along the dispersion curve. Thus we see that the coupled mode formalism is powerful enough to describe the behavior of the photonic crystal waveguide even for large index contrasts by using an empirical coupling consant implying that the only correction needed is in Eq. 24 and using a more exact form for Eq. 8 could lead to better quantitative predictions.

The usefulness of the coupled mode theory becomes more apparent when we consider the propagation loss of the waveguide. We can extract the propagation loss from the FDTD simulation as follows. Substituting a complex $\beta$ in Eq. 2, we have

$$
|E(\mathbf{r}, t)|^{2}=e^{2 \beta_{I} z}|E(x)|^{2}=e^{\alpha z}|E(x)|^{2}
$$

From our FDTD simulations, we compute a $Q$ defined as

$$
Q \equiv \omega \frac{\mathcal{E}_{0}}{-\Delta P}
$$

where $\mathcal{E}_{0}$ represents the stored energy and $\Delta P=\Delta \mathcal{E} / \Delta t$ is the power dissipation. By assuming low loss, we can approximate the exponential $\exp (-\alpha z) \sim 1-\alpha z$ and get an expression for $\alpha$.

$$
\alpha=2 \beta_{I}=\frac{-\Delta \mathcal{E}}{\mathcal{E}_{0}} \frac{1}{L}=\frac{\omega}{Q} \frac{\Delta t}{L}=\frac{\omega}{Q v_{g}}
$$

where we have chosen to let $L / \Delta t$ be the group velocity, $v_{g}=(d \beta / d \omega)^{-1}$. The propagation loss for three waveguides with cladding thicknesses of 5, 10, and 20 unit cells is shown in Fig. 5. Each waveguide consists of the same GaAs/air photonic crystal cladding and only differ in the cladding thicknesses. The same empirical 

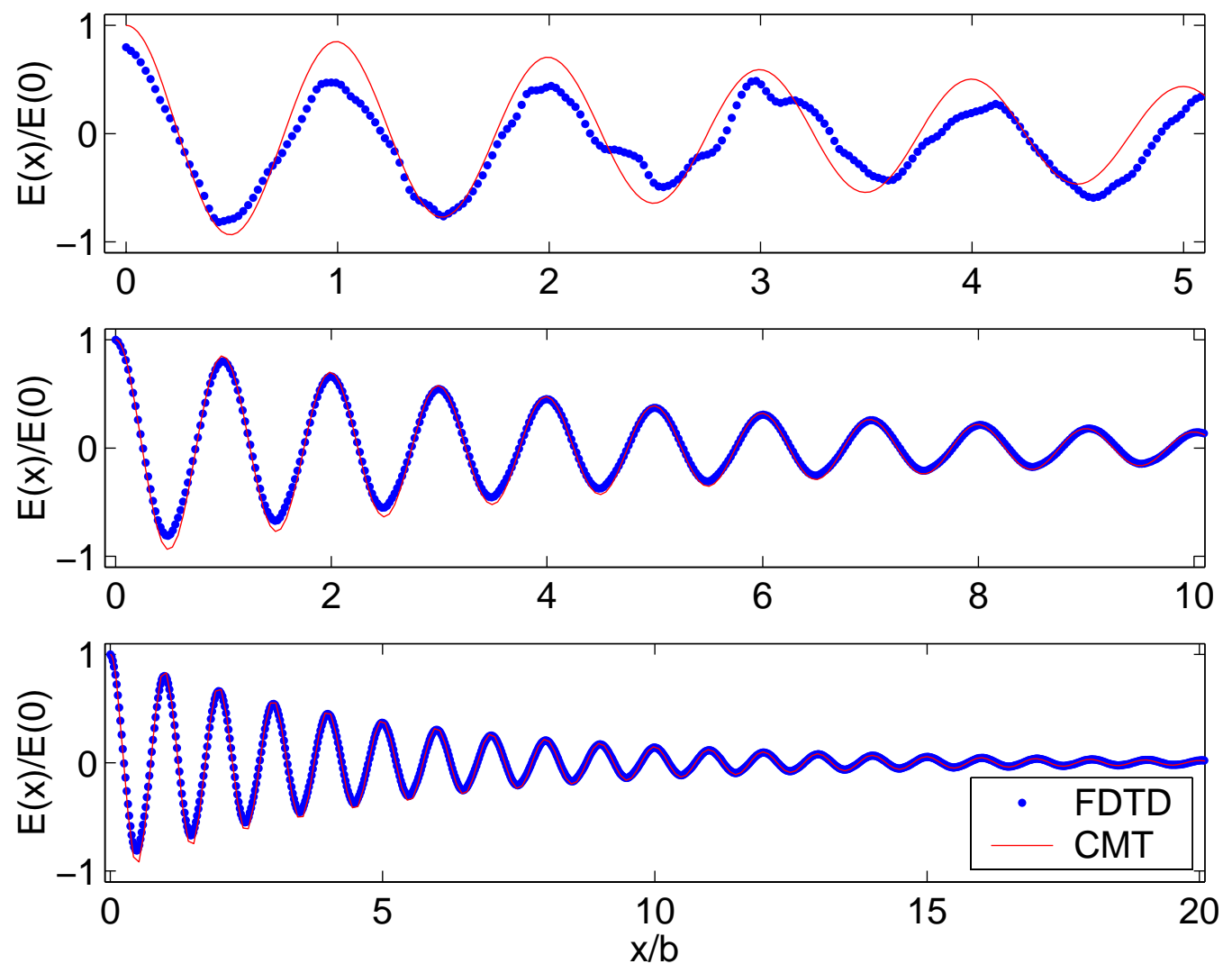

Figure 6. Transverse mode profiles of the three example waveguides analyzed for $\beta a / \pi=1 / 8$. The normalized field, $E(x) / E(0)$, is shown for waveguides with cladding thicknesses of 5,10 , and 20 unit cells.

coupling constant was used to calculate the loss for all three waveguides. For small propagation constants, the coupled mode prediction agrees very closely to the 2D FDTD simulations. The deviation when $\beta a>0.5 \pi$ is due to the fact that at higher $\beta$ values, the approximation $\left|k_{0}\right| \sim\left|k_{b}\right|$ is no longer valid and the higher order Bragg reflections, which we have ignored, become important.

The normalized transverse mode profiles, $E(x) / E(0)$, from the coupled mode theory as well as the 2D FDTD simulations are shown in Fig. 6 for the three waveguides with cladding thicknesses of 5, 10, and 20 unit cells. The propagation constant for each of the waveguides was chosen to be $\beta a / \pi=1 / 8$. Again, we see that with the empirical $\kappa$, the coupled mode approach matches the FDTD results quite closely. Thus, we see that the intuitive model used to analyze the photonic crystal waveguide is indeed useful and gives very good prediction about the modal field distribution.

\section{CONCLUSIONS}

A coupled mode formalism was used to analyze a GaAs susbstrate, air hole photonic crystal waveguide. Although coupled mode theory is, strictly speaking, valid for small index contrasts, from Fig. 4 and 6, we see that the coupled mode theory is indeed capable of describing the behavior of the photonic crystal waveguide even when the index contrast is many orders of magnitude greater than normally required for the coupled mode theory to be valid. A single empirical coupling constant is able to predict the dispersion, loss, and transverse field distribution for waveguides with different cladding thicknesses. 


\section{ACKNOWLEDGMENTS}

The authors would like to thank AFOSR (H. Schlossberg), DARPA (R. Athale), and ONR (Y. S. Park) for their generous support of this work.

\section{REFERENCES}

1. E. Chow, S. Y. Lin, J. R. Wendt, S. G. Johnson, and J. D. Joannopoulos, "Quantitative analysis of bending efficiency in photonic-crystal waveguide bends at $\lambda=1.55 \mu m$," Opt. Lett. 26, pp. 286-288, 2001.

2. M. Lončar, D. Nedeljkovič, T. P. Pearsall, J. Vučković, A. Scherer, S. Kuchinsky, and D. C. Allan, "Experimental and theoretical confirmation of Bloch-mode light propagation in planar photonic crystal waveguides," Appl. Phys. Lett. 80, pp. 1689-1691, 2002.

3. A. Chutinan and S. Noda, "Waveguides and waveguide bends in two-dimensional photonic crystal slabs," Phys. Rev. B 62, pp. 4488-4492, 2000.

4. M. Notomi, A. Shinya, K. Yamada, J. Takahashi, C. Takahashi, and I. Yokohama, "Structural tuning of guiding modes of line-defect waveguides of silicon-on-insulator photonic crystal slabs," IEEE J. Quantum Electronics 38, pp. 736-742, 2002.

5. K. S. Yee, "Numerical solution of initial boundary value problems involving Maxwell's equations in isotropic media," IEEE T Antenn Propag AP14, pp. 302-307, 1966.

6. K. M. Ho, C. T. Chan, and C. M. Soukoulis, "Existence of a photonic gap in periodic dielectric structures," Phys. Rev. Lett. 65, pp. 3152-3155, 1990.

7. K. M. Leung and Y. F. Liu, "Full vector wave calculation of photonic band structures in face-centered-cubic dielectric media," Phys. Rev. Lett. 65, pp. 2646-2649, 1990.

8. Z. Zhang and S. Satpathy, "Electromagnetic-wave propagation in periodic structures - Bloch wave solution of Maxwell equations," Phys. Rev. Lett. 65, pp. 2650-2653, 1990.

9. A. Yariv, "Coupled-wave formalism for optical waveguiding by transverse Bragg reflection," Opt. Lett. 27, pp. 936-938, 2002.

10. A. Yariv, Y. Xu, and S. Mookherjea, "Transverse Bragg resonance (TBR) laser amplifier." Opt. Lett. , To be published Feb. 2003.

11. A. Adibi, Y. Xu, R. K. Lee, A. Yariv, and A. Scherer, "Guiding mechanisms in dielectric-core photoniccrystal optical waveguides," Phys. Rev. B 64, p. 033308, 2001.

12. A. Adibi, Y. Xu, R. K. Lee, M. Lončar, A. Yariv, and A. Scherer, "Role of distributed Bragg reflection in photonic-crystal optical waveguides," Phys. Rev. B 64, p. 041102, 2001.

13. S. D. Gedney, "An anisotropic perfectly matched layer-absorbing medium for the truncation of FDTD lattices," IEEE Trans. Antennas Propag. 44, pp. 1630-1639, 1996. 